\title{
Risk Analysis of Tyramine Concentration in Food Production
}

\author{
L. Doudová*, F. Buňka ${ }^{\dagger}$ J. Michálek**, M. Sedlačík* and L. Buňková ${ }^{\dagger}$ \\ ${ }^{*}$ University of Defence, FEM, Kounicova 65, 66210 Brno,Czech Republic \\ ${ }^{\dagger}$ Tomas Bata University in Zlín, FT, Náměstí T. G. Masaryka 275, 76272 Zlín, Czech Republic \\ ** Brno University of Technology, FME, Technická 2896/2, 61669 Brno, Czech Republic
}

\begin{abstract}
The contribution is focused on risk analysis in food microbiology. This paper evaluates the effect of selected factors on tyramine production in bacterial strains of Lactococcus genus which were assigned as tyramine producers. Tyramine is a biogenic amine sythesized from an amino acid called tyrosine. It can be found in certain foodstuffs (often in cheese), and can cause a pseudo-response in sensitive individuals. The above-mentioned bacteria are commonly used in the biotechnological process of cheese production as starter cultures. The levels of factors were chosen with respect to the conditions which can occur in this technological process. To describe and compare tyramine production in chosen microorganisms, generalized regression models were applied. Tyramine production was modelled by Gompertz curves according to the selected factors (the lactose concentration of $0-1 \% \mathrm{w} / \mathrm{v}, \mathrm{NaCl} 0-2 \% \mathrm{w} / \mathrm{v}$ and aero/anaerobiosis) for 3 different types of bacterial cultivation. Moreover, estimates of model parameters were calculated and tested; multiple comparisons were discussed as well. The aim of this paper is to find a combination of factors leading to a similar tyramine production level.
\end{abstract}

Keywords: Gompertz curve, yield factor, multiple comparisons, tyramine production

PACS: $02.50 .-\mathrm{r}, 62.20 . \mathrm{fg}$

\section{INTRODUCTION}

Tyramine (TYM) belongs to a group of biogenic amines, which are alkaline compounds formed in foodstuff mainly by microbial decarboxylation. Biogenic amine (BA) precursors are free amino acids (tyrosine is a precursor for tyramine synthesis) provided by proteolytic changes of proteins and/or peptides (see [1]). Contaminating bacteria from family Enterobacteriaceae and genera Pseudomonas are usually included in groups of microorganisms possessing decarboxylase enzymes. TYM is produced also by some lactic acid bacteria (e.g. strains from genera Lactococcus, Lactobacillus, Enterococcus etc.). Lactic acid bacteria (LAB) also occur in some food as contaminants threatening the quality and safety of the product (e. g. in certain meat products, beer, and wine). Presence of particular decarboxylases is not specific within the species and occurs only among certain number of strains in the species (see [2], [3]).

TYM is an endogenous compound with key functions in metabolism of living organisms. Generally, low concentrations of TYM in food and beverage (basically under $100 \mathrm{mg} / \mathrm{kg}$ ) do not represent a significant risk for a healthy person. Human intestinal tract has a detoxifying system. However, higher amounts of BAs (generally above $100 \mathrm{mg} / \mathrm{kg}$ ) may induce undesirable psychoactive and vasoactive effects (hypotension or hypertension, headache, nausea, breathing problems etc.). Moreover, effectiveness of the detoxication system can also be diminished by antihistamines, antidepressants and alcohol. Due to the interference of alcohol with the detoxication metabolism, a lower TYM limit is usually declared for alcoholic beverages: $25-40 \mathrm{mg} / \mathrm{L}$ for TYM (see [1], [4]).

Production of biogenic amines (including TYM) by bacteria can be influenced by many external factors (temperature and $\mathrm{pH}$ of the environment, aero/anaerobiosis, availability of carbon sources, presence of growth factors, growth phase of the cells, $\mathrm{NaCl}$ concentration etc.), which can affect mainly the kinetics of decarboxylase reactions (see [1], [2], [5]). Biogenic amines can also be produced by LAB strains which are commonly used for technological purposes as starter cultures [2]. It would be suitable to describe the kinetics of biogenic amine production under conditions which are similar to those during the technological process of cheesemaking. However, such information occurs very rarely in current specialized literature [2], [5].

\section{PROBLEM DELINEATION AND APPLIED STATISTICAL METHODS}

The aim of the work was to model the effect of selected factors (the concentration of lactose in the range of $0-1 \%$ $\mathrm{w} / \mathrm{v}, \mathrm{NaCl}$ in the range of $0-2 \% \mathrm{w} / \mathrm{v}$ and an aerobic/anaerobic environment) on TYM production in one bacterial 
strain of Lactococcus lactis subsp. lactis (001) and two bacterial strains of Lactococcus lactis subsp. cremoris (002, 003) which are tyramine-positive. These bacteria are used within the biotechnological process of cheese production as starter cultures. Therefore, the levels of factors were chosen with respect to the conditions which can occur within the technological process. To describe and compare TYM production for chosen microorganisms, nonlinear regression models were applied.

TYM production has been modelled by Gompertz curves according to selected factors. Parameterization of Gompertz's curve was carried out considering [2], [5], and it was used in the form of

$$
y=\alpha \exp \left\{-\exp \left[\frac{\mu \mathrm{e}}{\alpha}(\lambda-t)+1\right]\right\},
$$

where $y$ is the tyramine content $(\mathrm{mg} / \mathrm{L}) ; \mu$ is the tyramine production rate $(\mathrm{mg}$ tyramine/L h); $\alpha$ is the asymptote defined as maximum tyramine production; $\lambda$ is the delay period (the time until the tyramine production was detected for the first time (hours)); $t$ is time (hours). Parameter estimates were carried out by nonlinear least squares; standard deviations of parameter estimates were determined, and in the course of statistical analysis, asymptotic normality of observed parameters was assumed (see [6] p. 24 Theorem 2.1). Multiple comparisons of parameters were performed by the Bonferroni method; again, asymptotic normality was assumed (see [7] p. 197 formula (5-27)).

Furthermore, the yield factor $\beta_{y}=Y_{T Y R / C F U}$ (where the colony-forming unit (CFU) is an estimate of viable bacterial numbers) was modelled using linear regression:

$$
y_{t}=y_{0}+\beta_{y}\left(N_{t}-N_{0}\right) \cdot 1000,
$$

where $y_{t}$ and $y_{0}(\mathrm{mg} / \mathrm{L})$ are the concentrations of tyramine at time $t$ and 0 , respectively; $N_{t}$ and $N_{0}$ are total numbers of $\mathrm{CFU} / \mathrm{mL}$ in time $t$ and 0 , respectively; and $\beta_{y}$ is a constant yield factor for tyramine formation. A test of equality between regression coefficients was transformed into testing of general linear hypothesis (see [7] p. 302); for test statistics, see [7] p. 303 formula (7-17).

Numerical calculations were obtained using the MATLAB 7.13. software.

\section{STATISTICAL ANALYSIS}

In order to assess the influence of four factors, i.e. environment (aerobic AE, anaerobic AN), lactose concentration LC $(0 \% ; 0.25 \% ; 0.5 \% ; 0.75 \% ; 1 \%) \mathrm{w} / \mathrm{v}$, concentration of $\mathrm{NaCl}(0 \%, 1 \%, 2 \%) \mathrm{w} / \mathrm{v}$ and lactic acid bacteria $(001,002,003)$ on the tyramine content $y(\mathrm{mg} / \mathrm{L}), 10$ observations were made for every variable $y$ (the tyramine content) depending on time (0-15 days), and for various combinations of individual factors. On that account, a total volume of $2 \times 5 \times 3 \times 3=90$ sets were available. Temporal progress of the $y$ variable was modelled for every possible combination of the four above-mentioned factors using the Gompertz curve. Consequently, the progress of tyramine $y_{t}$ was modelled using the $\beta_{y}$ yield factor depending on the absolute growth rate (CFU/L day).

While calculating estimates, the main attention was paid to the correct choice of initial estimates. Furthermore, variability of obtained estimates was considered. However, the number of observations in each group was not large (10 observations for each group of four studied factors), variability of estimates did not differ from usual limits. As an example, we present estimates of parameters $\alpha, \mu, \lambda$ and their standard deviations for Gompertz curve from figure 2 1. column and 2. row (bacteria 001, $\mathrm{AE}$ environment, $\mathrm{NaCl}$ concentration $1 \%, \mathrm{LC} 0.5 \%$ ), where the monitored points demonstrated greater fluctuation around the Gompertz curve. The values of obtained estimates and their standard deviations are $392.07 \pm 10.18$ for the parameter $\alpha, 155.13 \pm 24.24$ for the parameter $\mu$ and $1.40 \pm 0.21$ for the parameter $\lambda$.

The impact of environment on the tyramine content $y$ in a sample of lactic acid bacteria 001 with $1 \% \mathrm{NaCl}$ concentration and various lactose concentrations can be seen in the left graph in Figure 1. The figure illustrates the environment factor impact on individual curves well. It shows that for given values of other factors, the $y$ variable (tyramine content) is always higher in anaerobic environment. Similarly, the $\beta_{y}$ yield factor is always higher in AE environment than in AN environment. Both curves were compared by testing of equality of their parameters. It was proved that the environment factor is statistically significant at a significance level of $5 \%$. To be more precise, the hypothesis of $\alpha$ parameters equality, which describes maximum tyramine production, was rejected in AE and AN environment for all possible combinations of the remaining factors. Hypotheses about equality of $\mu$ parameters and equality of $\lambda$ parameters were rejected by statistical tests approximately in $50 \%$ of all cases. Tests on $\beta_{y}$ yield factor equality in $\mathrm{AE}$ and $\mathrm{AN}$ environment were not rejected only in case of the following combinations: bacteria 001, 


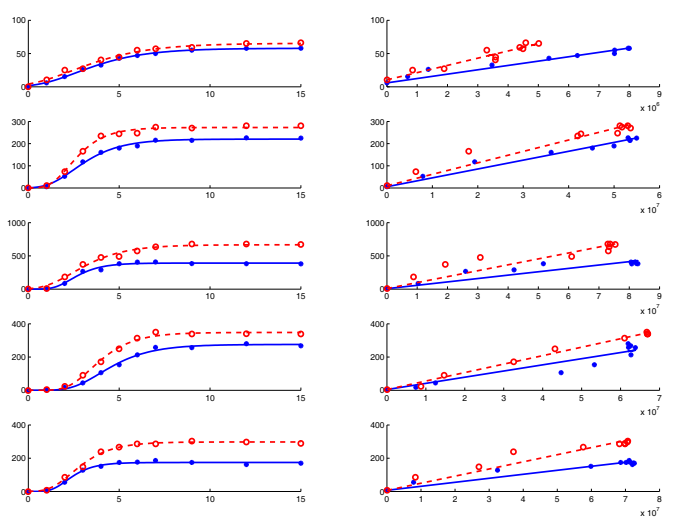

Left graph
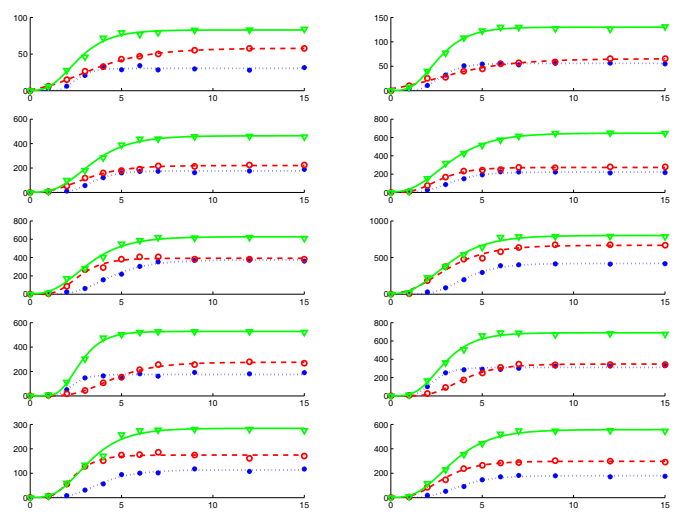

Right graph

FIGURE 1. Impact of $\mathrm{AE}$ (blue) and $\mathrm{AN}$ (red) environment (the left graph), and impact of $\mathrm{NaCl}(0 \%$ blue, $1 \%$ red, $2 \%$ green) (the right graph) on tyramine production. Left graph: bacteria $001, \mathrm{NaCl}$ concentration $1 \%$; Gompertz curves (first column), yield factor (second column); in rows, the CL gradually rises from $0 \%$ to $0.25 \% ; 0.5 \% ; 0.75 \% ; 1 \%$. Right graph: bacteria 001; AE environment is in the first column, $\mathrm{AN}$ in the second; in rows, the LC rises from $0 \%$ to $0.25 \% ; 0.5 \% ; 0.75 \% ; 1 \%$
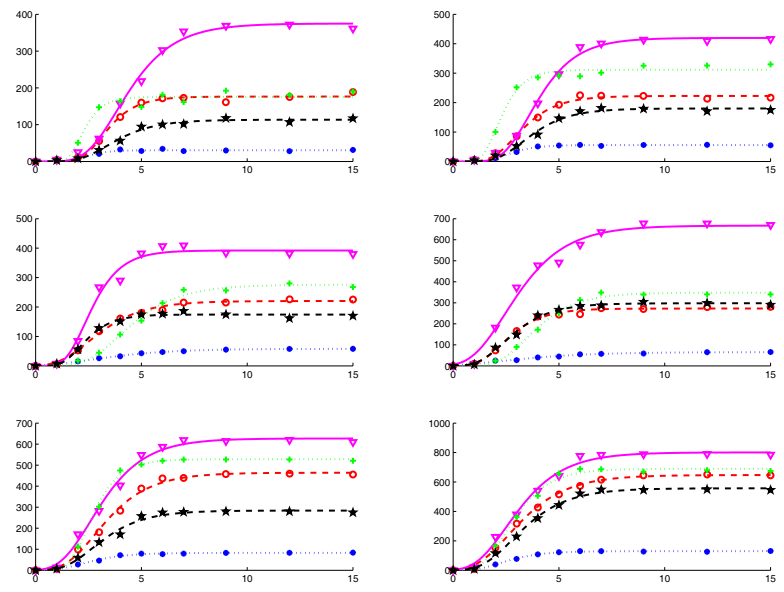

FIGURE 2. Impact of lactose concentracion( $0 \%$ blue, $0.25 \%$ red, $0.5 \%$ magneta, $0.75 \%$ green, $1 \%$ black) on tyramine production for bacteria 001. AE environment is in the first column, $\mathrm{AN}$ in the second; in rows the $\mathrm{NaCl}$ concentration gradually rises from $0 \%$ to $1 \% ; 2 \%$

$\mathrm{NaCl}$ concentration $0 \%, \mathrm{LC}=0.25 \%, 0.5 \%$; bacteria $002, \mathrm{NaCl}$ concentration $1 \%$ and $\mathrm{LC}=0.75 \%$; and bacteria $002, \mathrm{NaCl}$ concentration $2 \%$ and $\mathrm{LC}=1 \%$. For bacteria 003 , the hypothesis about yield factor $\beta_{y}$ equality was rejected for $\mathrm{NaCl}$ concentration $0 \%, 2 \%$ and for all LC levels; contrariwise, with $\mathrm{NaCl}$ concentration of $1 \%$, and $\mathrm{LC}=0.25 \% ; 0.5 \% ; 0.75 \% ; 1 \%$, equality of $\beta_{y}$ yield factor between $\mathrm{AE}$ and $\mathrm{AN}$ environment was not rejected.

The impact of $\mathrm{NaCl}$ concentration on tyramine production is well illustrated in the right graph in Figure 1 . Tests on $\alpha$ parameters (maximum tyramine production) equality for $\mathrm{NaCl}$ concentration $0 \%, 1 \%$, and $2 \%$ with given combinations of other factor values (bacteria, CL, environment) shows statistically significant differences at a significance level of $5 \%$ in all tested combinations. Values of $\alpha$ parameter (maximum tyramine production) are the highest when $\mathrm{NaCl}$ concentration is $2 \%$ and the lowest when $\mathrm{NaCl}$ concentration is $0 \%$. A similar situation occurred during comparison of yield factor $\beta_{y}$. It reaches its highest values when $\mathrm{NaCl}$ concentration is $2 \%$. With $\mathrm{NaCl}$ concentration $0 \%$ yield factor $\beta_{y}$ values were lower or the same as with $\mathrm{NaCl}$ concentration $1 \%$.

The impact of LC parameter (lactose concentration) is demonstrated in Figure 2. $\alpha$ parameters equality for various 
types of LC factor values (each time five $\alpha$ parameters were compared based on the LC level) in given combinations of other variable values were largely rejected. Values of $\alpha$ parameters were the highest when $\mathrm{LC}=0.5 \%$, and the lowest when $\mathrm{LC}=0 \%$. In case of yield factor $\beta_{y}$ comparison, results were different. Values of $\beta_{y}$ parameters were the highest when $\mathrm{LC}=0 \%$, and the lowest when $\mathrm{LC}=1 \%$. These differences are statistically significant at the level of significance of $5 \%$.

\section{DISCUSSION}

Higher production of TYM was observed in the environment with a higher concentration of $\mathrm{NaCl}$ in all strains tested. Pereira [8] suggested that $\mathrm{Na}^{+}$ions that are involved in regulation of intracellular $\mathrm{pH}$ play an essential role in the tyrosine decarboxylation pathway. $\mathrm{Na}^{+}$ions are important in sodium/proton antiport system, as they are exchanged with $\mathrm{H}^{+}$ions that are removed out of cells.

When lactose was not added to the medium, a lower growth of bacterial cells and a lower production of TYM (in all $\mathrm{NaCl}$ concentrations tested) were observed. A cultivation medium without lactose is less suitable for the tested lactococci, which need complex media for their growth. The highest amount of TYM was observed in the environment with $0.5 \%(\mathrm{w} / \mathrm{v})$ lactose. A further increase in lactose concentration, with the same $\mathrm{NaCl}$ concentration, led to a decreasing production of TYM. According to Molenaar [9], decarboxylation of amino acid may serve as a source of metabolic energy. Thus, in sub-optimal cultivation conditions, represented by medium without saccharide source of energy, i.e. lactose, the cells can obtain necessary energy also from decarboxylation processes. Therefore, the amount of TYM produced by a cell as a final product of this metabolic pathway increases. When lactose was added to cultivation broth, the yield factor $\left(Y_{T Y R / C F U}\right)$ decreased, probably because the cell could obtain energy more effectively from saccharides.

The last factor observed was an aerobic/anaerobic environment. In all strains tested under the given conditions, higher production of tyramine was determined under anaerobic conditions.

\section{ACKNOWLEDGMENTS}

The paper was supported by the "Application of Modified CPM on Security Criteria Portfolio in Logistics Chains" specific research programme.

\section{REFERENCES}

1. A. Halász, Á. Baráth, L. Simon-Sarkadi, and W. Holzapfel, Trends in Food Science and Technology 51, $42-49$ (1994).

2. L. Buňková, F. Buňka, M. Hlobilová, Z. Vaňátková, D. Nováková, and V. Dráb, European Food Research and Technology 229, 533-538 (2009).

3. P. Pleva, L. Buňková, A. Lauková, E. Lorencová, V. Kubáň, and F. Buňka, Veterinary Microbiology 159, $438-442$ (2012)

4. B. ten Brink, C. Damink, H. M. L. J. Joosten, and J. H. J. Huis in 't Veld, International Journal of Food Microbiology 11, 73-84 (1990).

5. L. Buňková, F. Buňka, E. Pollaková, T. Podešvová, and V. Dráb, International Journal of Food Microbiology 147, 112-119 (2011).

6. G. A. F. Seber, and C. J. Wild, Nonlinear Regression, John Wiley \& Sons, New York, 1989, pp. 24.

7. R. A. Johnson, and D. W. Wichern, Applied multivariate statistical analysis, Prentice-Hall, London, 1992, pp.197 and 302-303.

8. C. I. Pereira, D. Matos, M. V. San Romao, and M. T. B. Crespo, Applied and Environmental Microbiology 75, 345-352 (2009).

9. D. Molenaar, J. S. Bosscher, B. ten Brink, A. J. M. Driessen, and W. N. Konings, Journal of Bacteriology 175, 2864-2870 (1993). 